\title{
Agrobiodiversity Conservation to deal with Climate Change
}

\author{
S. Nepal
}

C imate change is real and the effects of climate change vary with different spatial scales. At a broader global scale, climate change may have negligible impact whereas at the local and regional scales, the impact may be severe and substantial. For instance, at the local/regional levels of an agricultural ecosystem, climate change may impact agricultural sustainability in two interrelated ways. First, it may reduce the long-term ability of the agroecosystem to provide enough food and fiber for the world's population and second, it may induce alteration in agro-climatic conditions and spatially shift agroecosystems, thus endangering natural habitats and their floral and faunal diversities. Climate change is, therefore, a serious concern for agricultural ecosystems and there is a need for an agrobiodiversity conservation approach to combat with these problems.

Agrobiodiversity comprises of all the components of biological diversity pertinent to food and agriculture present in agro-ecosystems, including microbes, insect pollinators, and the hedgerows that support soil stability and provide a home for wildlife. It also provides opportunities to develop and implement adaptation strategies to the biotic and abiotic stresses resulting from climate change, while mitigating the emissions of green house gases. So, agrobiodiversity can be the basis for reducing emissions of green house gases, sequestering carbon, suppressing pests and diseases, using water and nutrients efficiently and maintaining the productivity of agriculture as a whole. Thus in the long run, agrobiodiversity can be the basis for enhancing food security, livelihood, and the conservation of resource base to mitigate climate change.

Agrobiodiversity based conservation approaches to address the climate change include: i) genetic enhancement of crops for a more changing and heterogeneous environment, ii) use of integrated pest management (IPM) with the best combination of cultural, biological and technological measures of pest control, iii) change in cropping patterns by promoting agroforestry, agroenergy and new crop species, and iv)organic and traditional farming systems with less dependence on energy-intensive fertilizers, chemicals, and concentrated feeds.

Recently, a new concept of Agrobiodiversity Conservation Credit (ACC) has been proposed to address climate change. Climate change might consider exploring credits for carbon sequestration in soils through conservation tillage as well as agroforestry and other climate friendly practices (no use of fertilizers/pesticides) in agricultural landscapes. Compared to orthodox methods of agriculture, these approaches offer higher productivity per unit area, easy management for agricultural systems and less harm to human health and environment.

All these approaches have the potentials to address climate change. However, the most challenging job to implement these approaches is to convince people to accept these approaches. People's participation at various levels of planning and policy making may be effective for addressing these people. A key to address climate change may be increasing participation of the general public, private sectors, policy makers and research institutions to integrate agrobiodiversity research in various national and international agenda and plans to revitalize conservation efforts in agrobiodiversity.

In conclusion, climate change is real and inevitable; its effects cannot be completely controlled but large reductions of adverse impacts are possible when adaptation and mitigation measures are fully implemented. Agrobiodiversity conservation supported by integrated systems of environmental, social and political sciences is one of the best approaches to climate change and to influence multiple ecosystem services such as agricultural productivity, water quality, energy conservation and human health. 